\section{The ins and outs of enhancer validation}

genomic
integration
of candidate
enhancer
sequences
produced
results that
were more
reproducible
\[ \]

Comprehensive, functionally validated enhancer data sets for different tissues and cell types enable the systematic exploration of the roles of these regulatory elements in biology and disease. A new study by Inoue et al. now raises concerns about the validity of using episomal reporter assays to determine the functionality of candidate enhancers.

A caveat of existing approaches for the identification of candidate regulatory sequences in mammalian genomes is that enhancer elements are predicted indirectly; for example, based on antibody binding to epigenomic modifications or transcription factors in the case of chromatin immunoprecipitation followed by sequencing (ChIP-seq). Functional validation is often done using

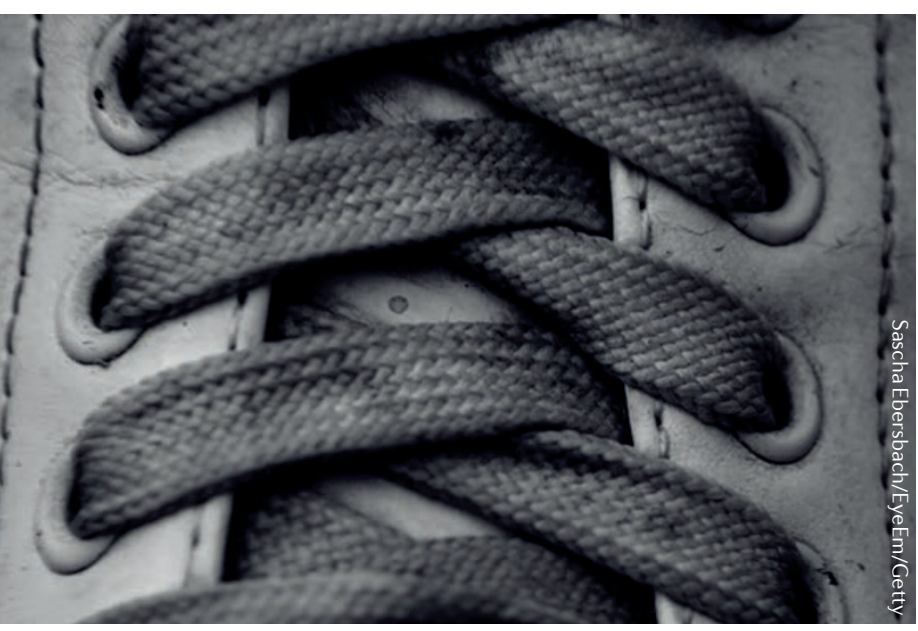

episomal reporter assays, in which a candidate enhancer sequence is inserted next to a minimal promoter that drives expression of a reporter gene and tested in an episomal manner (that is, without integrating into the genome). Multiplexing of these reporter assays by sequencing-based quantification of reporter barcodes in massively parallel reporter assays (MPRAs) have made this approach high-throughput.

In a systematic investigation, Inoue et al. set out to determine to what extent the episomal context in reporter assays can confound the functional validation of candidate enhancers. To this end, the functional activities of 2,236 potential liver enhancer sequences and 204 control sequences were compared in a chromosomally integrated versus an episomal context.

Using a lentiviral approach, the candidate liver enhancer MPRA library was packaged with either a wild-type integrase or a mutant integrase. The wild-type protein induces the lentivirus-driven integration of the MPRA library into the genome. By contrast, the mutant protein produces a non-integrating lentivirus that allows transient transgene expression from non-integrated DNA, thus acting like an episomal vector.

The assayed transgene was flanked by antirepressor sequences to protect from site-of-integration effects, and 100 independent reporter barcode sequences were used per assayed enhancer to achieve a high-throughput, quantitative measurement of each candidate's regulatory potential. The team extracted RNA and DNA from both wild-type and mutant infections, and amplified barcodes before sequencing.

Overall, genomic integration of candidate enhancer sequences produced results that were more reproducible, robust and biologically relevant than those obtained in an episomal context. For example, using single-feature models, the authors assessed more than 400 genomic annotations, which are generated based on biochemical marks measured in a native context. These annotations correlated markedly better with and were significantly more predictive of wild-type experiments than with results from the mutant integrase assay, which reflects the episomal context.

Overall, the study shows that chromosomally integrated reporter assays better reflect endogenous enhancer activity than episomal reporter assays. Nonetheless, the authors acknowledge that even integrated reporter assays have limitations, as they assess candidate enhancer sequences outside of their native context, and urge caution when interpreting the results of any reporter assay.

Linda Koch

ORIGINAL ARTICLE Inoue, F. et al. A systematic comparison reveals substantial differences in chromosomal versus episomal encoding of enhancer activity. Genome Res. http://dx.doi.org/ 10.1101/gr.212092.116 (2016) 